\title{
Some patterns of fixation saccadic eye movements
}

KENNETH GAARDER, CLINICAL NEUROPHARMACOLOGY RESEARCH CENTER

NATIONAL INSTITUTE OF MENTAL HEALTH, SAINT ELIZABETHS HOSPITAL, WASHINGTON, D. C.

Fixation saccadic eye movements occur at a more rapid rate during non-alpha (aroused) intervals than during alpha (less aroused) intervals. Other specific patterns of eye movement are shown to be typical of individuals or to occur in periods combining visual fixation with auditory task instruction.

It has recently been shown that saccadic eye movements reflect a mechanism for the discontinuous input of visual information (Gaarder, 1966b). Considerable re-evaluation of visual phenomena will occur as the importance of this becomes realized. It is now possible to ask questions re-examining data acquired in earlier studies. One of these concerns the relationship between eye movements and arousal, while the other has to do with unusual patterns of eye movements observed. The first question can be simply studied by the simultaneous examination of eye movement recordings and EEG, using the presence or absence of alpha rhythm as an index of arousal. The second question involves the inspection of eye movement records for unique properties which would not be anticipated.

Method

In gathering data for a number of studies (Gaarder et al, 1964; Gaarder et al, 1966; Gaarder \& Silverman, in preparation) large amounts of simultaneous eye movement and electroencephalographic recordings have been collected. The eye movements have either been recorded by the mirror-on-contact lens technique which gives accurate and linear measurement of a restricted range of movement or by the infra-red corneal reflectance method which gives a less linear following of eye movement, but which is more readily adaptable to a large number of Ss and allows measurement of much larger movements. It should be noted that the important parameter of these studies, the occurrence or non-occurrence of a saccade, is equally well detected by either method.

The Ss studied have been normal volunteers (Gaarder et al, 1964; Gaarder et al, 1966) or schizophrenic patients (Gaarder \& Silverman, in preparation). The task has been to fixate a point on a uniform background for several minutes while eye movements were recorded. More detailed descriptions of the experiments are given in the papers cited. The records of EEG and eye movements were inspected and epochs of good quality selected. The EEG was divided into samples according to the criteria of presence of clearcut alpha and presence of clear-cut non-alpha. A given S's record tended to have plenty of one and less of the other. The longest possible samples (1 sec. or more) of the less prevalent condition were matched with large nearby samples of the more prevalent. At least $40 \mathrm{sec}$. of each condition were measured per S. The number of saccades occurring during each sample and the length of each sample were then tabulated and the non-alpha and alpha rate of saccades per second were calculated for each $\mathrm{S}$.

Results

Relationship of rate of saccades to alpha and non-alpha periods. Comparison of saccadic rates in alpha and non-alpha periods in 11 normal ss showed the rate of saccades to be greater for non-alpha periods in 10 of the Ss. The mean rate of increase was 0.24 saccades per sec. $(p<.01$, Wilcoxon, 1949, paired replicate test). Figure 1 is a scattergram comparing the rate of saccades during alpha periods with the rate of saccades during non-alpha periods. It can be seen that quite large increases occur in some cases, while the case with decrease is less marked. However, very large samples of that case continued to show decrease so the effect does not appear to be one of sampling error.

Unusual patterns of eye movements. Five of 11 normal Ss showed various amounts of a particular sequence of eye movements (Fig. 2) consisting of three saccades in alternate horizontal directions with the first pair separated by $50 \mathrm{msec}$, and the second pair separated by 100 to $300 \mathrm{msec}$. The one $S$ who showed the most of this sequence had this pattern for approximately half of the saccades occurring.

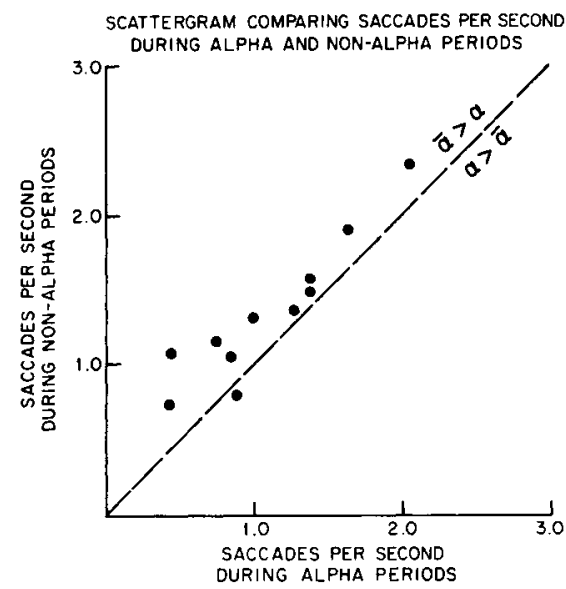

Fig. 1. Scattergram comparing rate of saccades during alpha periods with rate of saccades during non-alpha periods. Each point represents this comparison in one of 11 normal Ss. Diagonal line represents equal rate. Non-alpha rate is greater than alpha rate above the diagonal line and less under the line. 


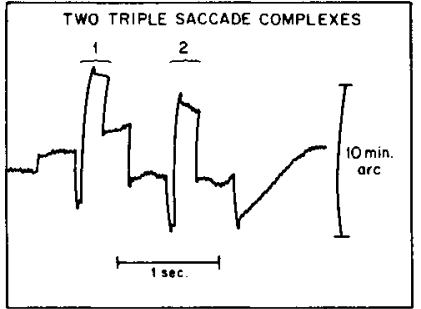

Fig. 2. Two triple saccadic groups (1 and 2) separated by a single saccade between. Recording by mirror-on-contact lens technique. First interval of $50 \mathrm{msec}$. followed by sec. of about 200 msec.

The other noteworthy pattern was seen in several schizophrenic and normal Ss. Its incidence could not be determined since it was not studied in the entire group. Here the eye movement pattern would be typical until the $\mathrm{S}$ was given task instructions about a stimulus to be flashed in the visual field. During the instructions the eye movements consistently changed to a rapidly alternating regular pattern (Fig. 3).

\section{Discussion}

The most important finding reported is the increase in rate of eye movements with absence of alpha. Since absence of alpha is considered to signify increased arousal, this means saccadic eye movements are increased with increased arousal. It would appear too simple to assume causality in either direction, however. Since there is reason for linking saccadic eye movements with alpha-linked periodicity (Gaarder et al, 1966), the increased rate of eye movements with arousal could represent either a shortened basic period or an increase of the frequency with which a period contained a saccade or both. An adequate model of the relationship will also have to explain the paradoxical case where rate of eye movement decreases with arousal. This could have been non-alpha representing decreased arousal if the alpha had been extinguished by sleepiness. In the most general sense, it has been shown that the rate of assimilating visual edge information tends to be increased during aroused states.

The unusual triple saccade eye movement pattern represents a unique method of data input presentation. In terms of a periodic discontinuous input model, it

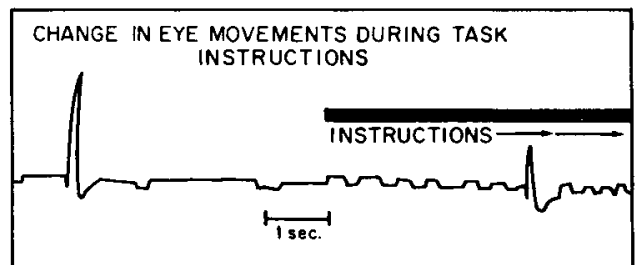

Fig. 3. Change of eye movement pattern during task instructions. Bar on right marks period of task instructions. Typical quasirandom intersaccadic intervals change to more rapid, more regular intervals during task instructions. means that during the sequence the S characteristically takes in two packets which would have to be more rapidly compared than is usual, and then after a more typical interval takes a third packet to compare to the resultant of the first two. This may be ultimately reconcilable with two-flash blanking experiments (Robinson, 1966). It must represent a unique visual style and hopefully individuals showing it can eventually be studied in other visual tests.

The pattern during task instructions is suggestive of a particular method of synchronizing visual input behavior with simultaneous auditory input behavior. Under the impact of the task of having to attend to auditory material in the form of task instructions, the visual input behavior (saccadic eye movements) becomes increased in rate and more regular to effect some sort of synchronization. This fits with Kristofferson's (1966) time shared data processing system model, in which the implication would be that the visual and auditory inputs were alternately programmed in a fixed sampling sequence. It would also, however, be compatible with other models which consider sensory interaction processes and with the simple idea of increased arousal during task instructions.

A major implication of the reported findings is that by considering visual input as discontinuous and then studying the discontinuities, one can learn more about vision. It has been shown earlier (Gaarder, 1966a) that during inattention saccades are replaced by sinusoidal instability drifts. Now the finding is added that the rate of saccades increases with arousal so that a correlated continuum is established of state of attention and arousal on the one hand and rate of eye movements on the other. Mulholland \& Evans (1966) consider the same correlation but draw causal inferences which I have not attempted. The unique patterns reported further imply that the study of perceptual styles and of sensory modality interactions might be undertaken with eye movement measurement techniques.

\section{References}

Gaarder, K. Fine eye movements during inattention. Nature, 1966a, $209,83$.

Gaarder, K. R. Transmission of edge information in the human visual system. Nature, $1966 \mathrm{~b}, 212,321-323$.

Gaarder, K., Krauskopf, J., Graf, V., Kropfl, W., \& Amington, J. C. Averaged brain activity following saccadic eye movements. Science, 1964, 146, 1481-1483.

Gaarder, K. R., Koresko, R. L., \& Kropfl, W. The phasic relation of a component of alpha rhythm to fixation saccadic eye movements. EEG clin. Neurophysiol., 1966, 21, 544-551.

Kristofferson, A. B. A quantal interpretation of sensory channel uncertainty and reaction time and the physiological time quantum and the discrimination of succession. NASA Contractor Report NASA CR-453. May, 1966.

Mulholland, T., \& Evans, C. R. Oculomotor function and the alpha activation cycle. Nature, 1966, 211, 1278-1279.

Robinson, D. N. Disinhibition of visually masked stimuli. Science, $1966,154,157-158$.

Wilcoxon, F. Some rapid approximate statistical procedures. American Cyanamid Co., New York, 1949. 